\title{
Mammary analogue secretory carcinoma of the parotid gland: a rare and unique entity
}

\begin{abstract}
Mammary analogue secretory carcinoma (MASC) of the salivary gland is a recently described, rare, and unique diagnostic entity. This unusual lesion corresponds histopathologically and cytogenetically to secretory carcinoma of the breast. MASC of the salivary gland displays overlapping features of both mammary secretory carcinoma and acinic cell carcinoma (AciCC). This tumor is characterized by the chromosomal translocation (12;15) (p13;q25), which results in the fusion gene ETV6-NTRK3. We report a rare case of MASC presenting as a left parotid mass in a 48year-old male, which was confirmed by ETV6 gene rearrangement studies by Fluorescence in situ hybridization (FISH). MASC, while rare, should be considered in the differential diagnosis of salivary gland lesions.
\end{abstract}

Keywords: mammary analogue secretory carcinoma, masc, parotid, parotid neoplasm, parotid tumor, salivary gland tumor, acinic cell carcinoma, head and neck cancer, parotidectomy
Volume 5 Issue 3 - 2016

\author{
Kenneth Rosenstein,' Amit Bhojwani,' \\ Melanie Silva, ${ }^{2}$ Darshan Roy, ${ }^{2}$ Alan \\ Shienbaum ${ }^{3}$ \\ I Department of Otolaryngology Head and Neck Surgery \\ Rowan University School of Osteopathic Medicine, USA \\ 2Rowan University School of Osteopathic Medicine, USA \\ 3Department of Pathology Rowan University School of \\ Osteopathic Medicine, USA
}

Correspondence: Kenneth Rosenstein Department of Otolaryngology Rowan University 42 East Laurel Road Ste 2600 Stratford NJ 08084, USA, Email kennethrosenstein@gmail.com

Received: November 14, 2016 | Published: December 30, 2016
Abbreviations: MASC, mammary analogue secretory carcinoma; AciCC, acinic cell carcinoma; FISH, fluorescence in situ hybridization; PORT, post-operative radiation therapy; ADC-NOS, adenocarcinoma not otherwise specified

\section{Introduction}

Mammary analogue secretory carcinoma (MASC) was first described by Skálová et al.,' in 2010 as a unique entity of the salivary glands. Its pathological features are consistent with the secretory carcinomas of the breast. ${ }^{1,2}$ MASC has a distribution across all age groups with a mean of 46years of age and predominance in young male patients. The parotid gland is the most common site of occurrence in $70 \%$ of cases. However, non-parotid sites consisting of serous acini including the submandibular, palatine, and labial glands have also been reported. MASC displays overlapping features of both mammary secretory carcinoma and acinic cell carcinoma (AciCC) and is composed of microcystic, cribiform, tubular, papillary, follicular solid or glandular growth patterns. ${ }^{3-6}$ Immunocytochemistry of MASC is positive for mammoglobin and S100. Diagnosis of MASC is confirmed with the detection of chromosomal translocation $(12 ; 15)$ (p13;q25) leading to the fusion gene ETV6-NTRK3..$^{4,6-8}$ As a result of this translocation, the tyrosine kinase is constitutively active. Additionally, the Ras-MAP kinase mitogenic and the PI-3 kinase-AKT pathways are likely activated as well. ${ }^{8}$ These pathways have been implicated in cancer and induce anti-apoptotic signals, thereby promoting tumor survival. ${ }^{9}$

Immunohistochemistry demonstrates positivity for S-100, mammaglobin, GCDFP-15, MUC1, GATA-binding protein 3, adipophilin, $\alpha$-amylase, DOG-1, SOX-10 and p63., ${ }^{1,50}$ Clinically, MASC usually portends a good prognosis; however, in some cases, high-grade transformation has been reported and poor outcomes have been demonstrated. ${ }^{6}$

\section{Case presentation}

A 48year-old male with a past medical history of Hodgkin lymphoma 27years ago presented to the clinic with a left parotid mass that had been growing slowly under observation for one year. CT scan of the neck demonstrated a small, well-circumscribed, contrast-enhancing mass in the superficial parenchyma of the left parotid gland (Figure 1). An ultrasound confirmed the $\mathrm{CT}$ findings, validating the superficial, well-circumscribed mass seen on CT (Figure 2). Fine-needle aspiration was performed and was inconclusive. In the case of MASC and AciCC, prior studies have shown that interpretation of aspirated tissue may be mistaken as normal salivary gland. ${ }^{2}$ Given the indefinite nature of the tumor, a left superficial parotidectomy was performed with preservation of the facial nerve. The mass was removed from the parenchyma of the parotid tail. Upon gross examination the encapsulated tumor was gray-brown in color, rubbery in appearance, and measured approximately 1.5 to $2 \mathrm{~cm}$ in diameter. No regional lymph node involvement was noted during surgery.

The lobulated mass contained nests of cytologically bland cells with microcystic, solid and acinar growth patterns and luminal eosinophilic secretions. Areas of the tumor showed an invasive appearance with stromal reaction and irregular growth pattern. Tumor was present at the resection margins.

Immunohistochemical staining was positive for S100, mammaglobin, and GCDFP15 (Figure 3). Fluorescence in situ hybridization (FISH) was positive for ETV6 gene rearrangement with the NTRK3 gene on chromosome 12 , confirming mammary analogue secretory carcinoma.

The patient in our report underwent a superficial parotidectomy with no post-operative complications noted. A skull-base to thigh PET-CT demonstrated FDG avidity (SUV 3.7) in a left submandibular node, level $1 \mathrm{~b}$, likely inflammatory in nature. The pathology, as described above, was consistent with MASC. No other findings were present. The patient had a positive resection margin, and as a result, the patient underwent radiotherapy with a total dose of $60 \mathrm{~Gy}$ divided into 30 fractions. During the course of radiation, the patient reported slight taste changes and minimal pain, both of which improved over the next two months. A follow-up PET-CT performed six months later was found to be negative for recurrent or persistent disease. Twelve months later, the patient remains disease-free. 

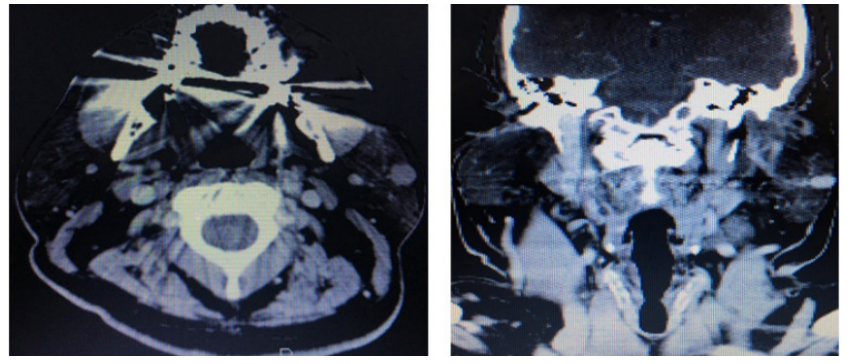

Figure I CT neck with contrast axial and coronal images, respectively, demonstrated a small, well-circumscribed, contrast-enhancing mass in the superficial parenchyma of the left parotid gland.

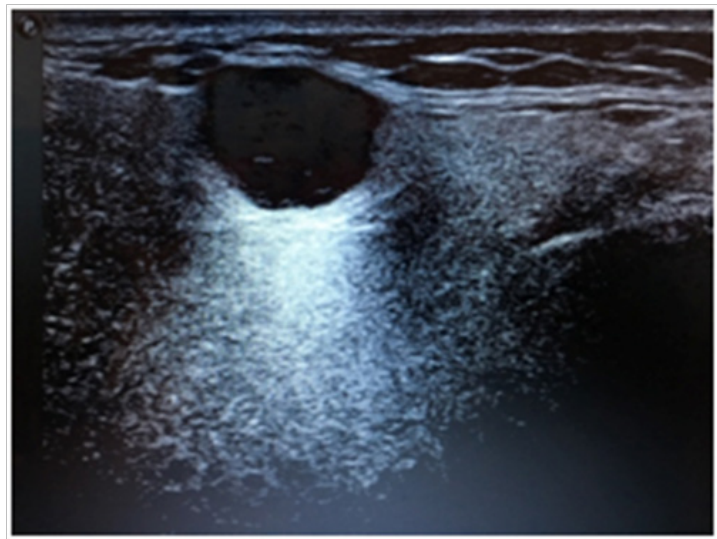

Figure 2 An ultrasound confirmed the CT findings, validating the superficial, well-circumscribed mass seen on CT. No suspicious features were identified on this image.

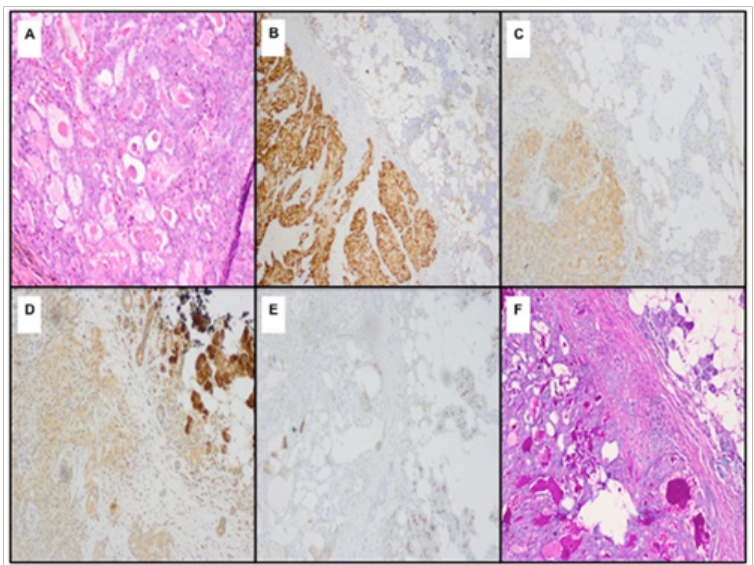

Figure 3 H\&E sections (A) show a tumor composed of small glands with luminal secretions with the tumor cells being positive for SI00. (B) and (C) demonstrate positive mammaglobin staining. (D) demonstrates positive GCDFP-I5 staining. In (E), the tumor cells are negative for DOGI. Lastly, (F) shows that the luminal secretions are positive for PAS.

\section{Discussion}

Skálová et al., ${ }^{1}$ have been following this entity closely and have stated that there have been a low number of cases of MASC with subsequent follow-up, treatment data, and an understanding of prognosis. ${ }^{6}$ Over the last six years, many studies have discerned the clinical behavior and prognosis of this rare and unusual malignancy. MASC has a propensity for high-grade transformation, which has previously been reported as an occurrence seen in AciCC. ${ }^{6}$ When high-grade transformation is observed, patients tend to be significantly older, and the likelihood for angiolymphatic invasion and metastatic disease is more common, thus portending a poorer prognosis. ${ }^{1,6,8,11}$ Furthermore, MASC also demonstrates greater regional lymph node involvement than does AciCC ${ }^{6,8,12}$. The presence of a high-grade lesion will thus alter staging, prognosis, and treatment. ${ }^{6}$

Low-grade salivary gland malignancies, such as MASC, are usually managed with post-operative radiation therapy (PORT) for the following indications: positive margins, perineural invasion, or the patient has a T3 or T4 tumor. ${ }^{8,13}$ An analysis from a cohort consisting of patients with mixed salivary gland neoplasms showed that PORT was most effective in controlling loco-regional recurrence in patients with incomplete resection or advanced T stage. ${ }^{8,13}$ A PORT dose of at least $60 \mathrm{~Gy}$ for the aforementioned indications should be used. ${ }^{13}$

The differential diagnosis includes acinic cell carcinoma, mucoepidermoid carcinoma, adenocarcinoma, not otherwise specified (ADC-NOS), low-grade cribriform cystadenocarcinoma of the salivary gland. ${ }^{5,8}$ As evidenced in the literature, the clinical behavior of this neoplasm varies significantly, from a slow-growing mass, metastatic disease at presentation, to death. ${ }^{8}$ Thus, the clinician should be prudent in establishing an early diagnosis and proceeding with surgery and/or radiation in an expeditious manner.

\section{Conclusion}

MASC of the salivary gland is a unique entity that should be considered on the differential diagnosis of a salivary gland lesion. Moreover, an FNA of this lesion may be inconclusive. Diagnostic features include a $(12 ; 15)(\mathrm{p} 13 ; \mathrm{q} 25)$ chromosomal translocation resulting in the ETV6-NTRK3 fusion gene, as well as positive immunohistochemistry for S-100, mammaglobin, and GCDFP-15. Although MASC shares overlapping features of breast mammary secretory carcinoma and acinic cell carcinoma, it can undergo high-grade transformation and may have greater regional lymph node involvement than AciCC, contributing to its poorer prognosis. Thus, differentiating between the various salivary gland lesions early in the disease course is essential in providing an accurate diagnosis and timely care.

\section{Acknowledgments}

None.

\section{Conflicts of interest}

Author declares there are no conflicts of interest.

\section{Funding}

None.

\section{References}

1. Skalova A, Vanecek T, Sima R, et al. Mammary analogue secretory carcinoma of salivary glands, containing the ETV6-NTRK3 fusion gene: a hitherto undescribed salivary gland tumor entity. Am J Surg Pathol. 2010;34(5):599-608.

2. Griffith CC, Stelow EB, Saqi A, et al. The cytological features of mammary analogue secretory carcinoma: a series of 6 molecularly confirmed cases. Cancer Cytopathol. 20 13;121(5):234-241.

3. Jin S, Ma H, He Y. Preservation of Facial Nerve with Adjuvant Radiotherapy for Recurrent Mammary Analogue Secretory Carcinoma of Parotid Gland. J Craniofac Surg. 2016;27(4):e364-e366.

4. Salat H, Mumtaz R, Ikram M, et al. Mammary Analogue Secretory Carcinoma of the Parotid Gland: A Third World Country Perspective-A Case Series. Case Rep Otolaryngol. 2015;2015:697254. 
5. Damjanov I, Skenderi F, Vranic S. Mammary Analogue Secretory Carcinoma (MASC) of the salivary gland: A new tumor entity. Bosn J Basic Med Sci. 2016;16(3):237-238.

6. Skalova A, Vanecek T, Majewska H, et al. Mammary analogue secretory carcinoma of salivary glands with high-grade transformation: report of 3 cases with the ETV6-NTRK3 gene fusion and analysis of TP53, betacatenin, EGFR, and CCND1 genes. Am J Surg Pathol. 2014;38(1):23-33.

7. Serrano-Arevalo ML, Mosqueda-Taylor A, Dominguez-Malagon H, et al. Mammary analogue secretory carcinoma (MASC) of salivary gland in four Mexican patients. Med Oral Patol Oral Cir Bucal. 2015;20(1):e23-e29.

8. Sethi R, Kozin E, Remenschneider A, et al. Mammary analogue secretory carcinoma: update on a new diagnosis of salivary gland malignancy. Laryngoscope. 2014;124(1):188-195.

9. Chalhoub N, Baker SJ. PTEN and the PI3-kinase pathway in cancer. Annu Rev Pathol. 2009;4:127-150.
10. Stevens TM, Kovalovsky AO, Velosa C, et al. Mammary analog secretory carcinoma, low-grade salivary duct carcinoma, and mimickers: a comparative study. Mod Pathol. 2015;28(8):1084-1100.

11. Chintakuntlawar AV, Shon W, Erickson-Johnson M, et al. High-grade transformation of acinic cell carcinoma: an inadequately treated entity? Oral Surg Oral Med Oral Pathol Oral Radiol. 2016;121(5):542-549.

12. Chiosea SI, Griffith C, Assaad A, et al. Clinicopathological characterization of mammary analogue secretory carcinoma of salivary glands. Histopathology. 2012;61(3):387-394.

13. Terhaard $\mathrm{CH}$, Lubsen $\mathrm{H}$, Rasch $\mathrm{CR}$, et al. The role of radiotherapy in the treatment of malignant salivary gland tumors. Int J Radiat Oncol Biol Phys. 2005;61(1):103-111. 